\title{
Noise Reduction for Digital Communications- The Masterpiece, a Modified Costas Loop
}

\author{
János Ladvánszky \\ Retired from Ericsson Hungary, Budapest, Hungary \\ Email: Ladvanszky55@t-online.hu
}

How to cite this paper: Ladvánszky, J. (2020) Noise Reduction for Digital Communications-The Masterpiece, a Modified Costas Loop. Circuits and Systems, 11, 57-64.

https://doi.org/10.4236/cs.2020.116006

Received: February 14, 2020

Accepted: June 25, 2020

Published: June 28, 2020

Copyright (อ 2020 by author(s) and Scientific Research Publishing Inc. This work is licensed under the Creative Commons Attribution International License (CC BY 4.0).

http://creativecommons.org/licenses/by/4.0/ (c) (i) Open Access

\begin{abstract}
An efficient way of noise reduction has been presented: A modified Costas loop called as Masterpiece. The basic version of the Costas loop has been developed for SSB SC demodulation, but the same circuit can be applied for QAM (quadrature amplitude modulation) demodulation as well. Noise sensitivity of the basic version has been decreased. One trick is the transformation of the real channel input into complex signal, the other one is the application of our folding algorithm. The result is that the Masterpiece provides a 4QAM symbol error rate (SER) of $6 \times 10^{-4}$ for input signal to noise ratio (SNR) of -1 $\mathrm{dB}$. In this paper, an improved version of the original Masterpiece is introduced. The complex channel input signal is normalized, and rotational average is applied. The $4 \mathrm{QAM}$ result is SER of $3 \times 10^{-4}$ for SNR of $-1 \mathrm{~dB}$. At SNR of $0 \mathrm{~dB}$, the improved version produces 100 times better SER than that the original Costas loop does. In our times, this topic has a special importance because by application of our Masterpiece, all dangerous field strengths from $5 \mathrm{G}$ and $\mathrm{WiFi}$, could be decreased by orders of magnitude. The Masterpiece can break the Shannon formula.
\end{abstract}

\section{Keywords}

Noise, Symbol Error Rate, QAM, Costas Loop, Hilbert Filter, Folding Algorithm

\section{Introduction}

Noise reduction is an important problem in communications. Digital communications are also sensitive to the noise. Effect of the noise can be detected by the symbol error rate (SER) as a function of signal to noise ratio (SNR). A possible circuit for noise reduction in digital communications is the Costas loop [1] whose original version has been developed for SSB SC demodulation. Essentially the same version can be used for 4QAM (Figure 1). 
Costas loop has been formulated from the phase locked loop (PLL, Figure 2) [1] with introduction of separate branches for I and Q signals. A combination of the I and Q signals is used as VCO driving signal, and the two mixers have been supplied by the same VCO output signal and its phase shifted version, respectively. To understand the details of operation and its analytical treatment, please refer to [2].

The problem is that the Costas loop version in Figure 1 is noise sensitive. Several tricks can be applied to decrease its noise sensitivity. Here we list them and apply some of them simultaneously.

\subsection{Complex Costas Loop}

Real Costas loop is known primarily for SSB demodulation. Complex Costas loop is intended basically for QAM demodulation. From the real input signal, an analytical complex signal is formulated using Hilbert filter. Similarly, analytical version of the VCO signal is formulated. Accordingly, Complex Costas loop comprises a complex mixer and VCO signal also should be complex. In other respects, structure is the same as that for real Costas loop. Basic advantages are that BER can be better at the same value of SNR.

\subsection{Averaging Method}

This is a method for stopping the rotation of the constellation diagram. In the VCO drive branch, signal is averaged in parallel using two different time constants. If the results are the same, then the constellation diagram stops rotation.

\subsection{4th Power Method}

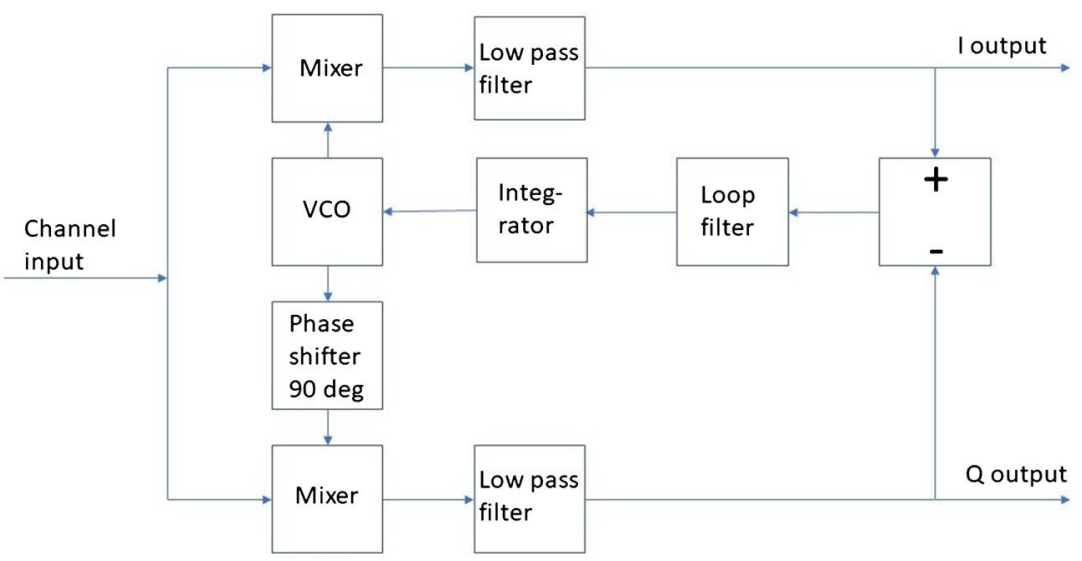

Figure 1. Costas loop for 4QAM demodulation.

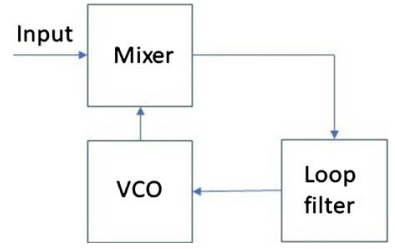

Figure 2. Phase locked loop. The VCO output phase is related to the phase of the input signal. A simple modification can be used for frequency multiplication. 
Used for carrier recovery of 4 QAM. If the receiver input signal is raised to the $4^{\text {th }}$ power, then the four constellation points are transformed into the same point. That means, in one step, all information has been removed but the carrier. Advantage is very exact reproduction of the carrier. Noise sensitive.

\subsection{Pulse Counting Method}

For stopping rotation of the constellation diagram, horizontal and vertical projections of the rotating constellation diagram contain extra steps compared to the case without rotation. Making pulses from steps by differentiation and counting and minimizing the number of steps, can be used for stopping rotation.

\subsection{Folding Method}

The folding method is very much noise insensitive. It replaces 4 th power method. Constellation diagram is folded along an axis then the result is shifted into a symmetric position with respect to the origin. This step is repeated until one point (the carrier) remains. This method can be used for real Costas loop as well, and for QAM of arbitrary degree. BER of 0.01 is possible at SNR of $-4 \mathrm{~dB}$.

\subsection{Normalization}

Used before correlation, complex signal is normalized exploiting that exp(jwt) has an absolute value of 1 . It cannot be used for real signal.

\subsection{Limitation of the VCO Drive Signal}

It is used for stopping rotation, especially in large noise. We observed that adding a large noise to the useful signal at the input of the Costas loop, significantly increases VCO drive signal thus causing rotation. Limitation of the VCO signal from below and above, limits the effect of the noise on the VCO signal.

\subsection{QAM SC}

It is observed that carrier in the receiver input signal interferes with the carrier produced by the Costas loop. Thus carrier (and possibly one sideband) at the receiver input has been removed by a filter.

\subsection{Correlation Method}

Used for stopping rotation. QAM signal is produced in two different ways and the results are correlated. Deviation of the correlation coefficient from 1 is used as VCO drive signal.

\subsection{Differential Coding}

Used for stopping rotation. Differential coding is not affected by rotation. We code the modulation signal with differential coding, and after demodulation, we use the same code for decoding [3].

Our intention is to find a method for noise reduction that is better than the 
previously known ones. From the above list, we combine application of complex input signals (Section 2), the folding algorithm (Section 3), and application of rotational average (Section 4). In Section 5 we show that by combination of these methods, exceptional insensitivity against noise can be achieved.

\section{Application of Complex Input Signals}

Basic version of the Costas loop is changed by inserting a block between the channel and the input of the Costas loop [2] (Figure 3). Essence of the change is application of complex signals [2]. However, in [2], the advantages are not fully exploited. We add normalization of the input signal, which has a significant effect on noise reduction.

It is widely known that in order to produce an analytic signal, imaginary part of the signal can be formulated by application of a Hilbert filter for the real signal [1]. Narrow-band approximation of a Hilbert filter is a 90 deg phase shifter or the corresponding delay circuit.

To remove a part of the noise from the complex signal, it is normalized by setting its absolute value to unity. Effect of application of a complex signal and its normalization has been shown in Figure 4.

Because of insertion of the block into the Costas loop, a complex mixer must

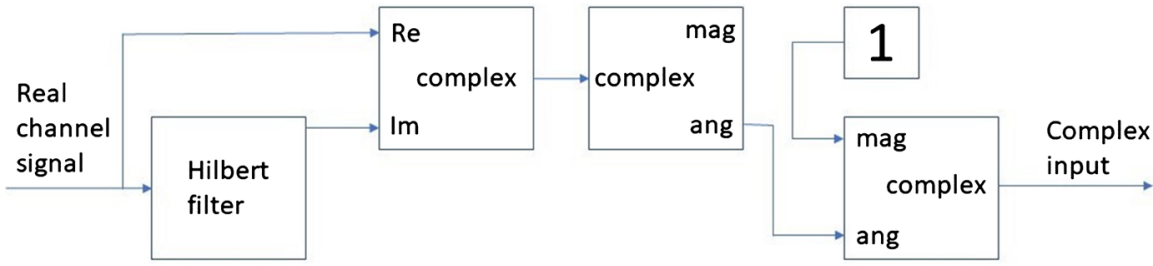

Figure 3. Transformation of the real channel signal into a complex signal and its normalization.

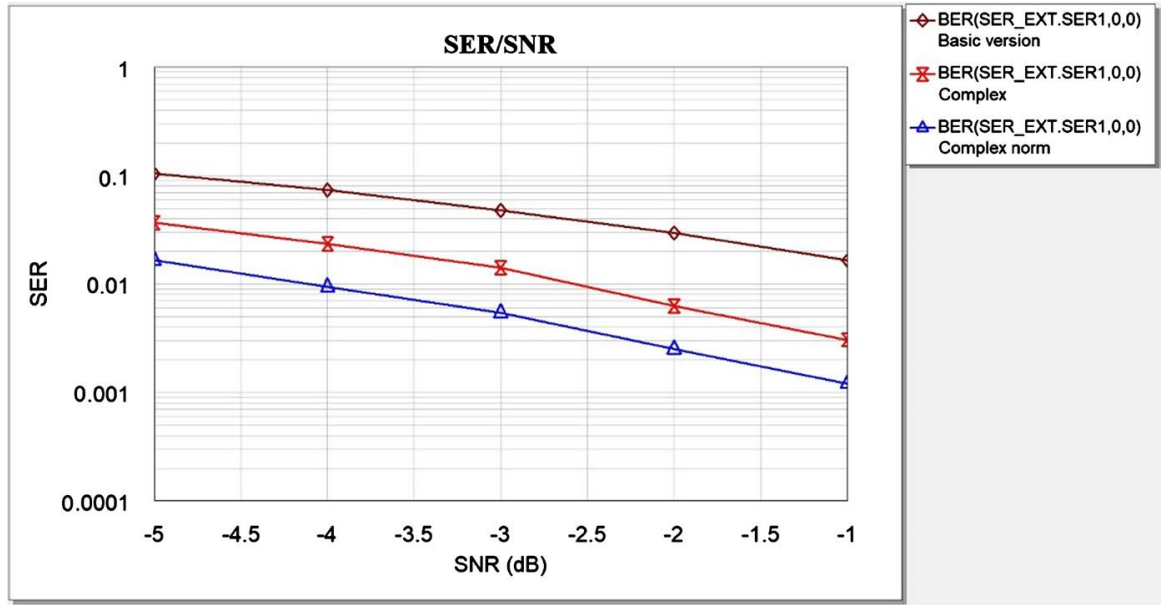

Figure 4. Effect of application of a complex signal and its normalization on noise properties. Three curves for SER vs. SNR are shown. The upper curve is without complex signal. The middle curve is with complex signal but without normalization. Bottom curve is with normalization. 
be used instead of the two real mixers, the VCO signal must also be complex and there is a modification at the beginning of the branches. We detail these modifications in Section 5.

\section{The Folding Algorithm}

Folding algorithm [4] means folding for 4QAM constellation diagram twice, one across the real axis and another one across the imaginary axis (Figures 5-7). As the noise is different around all points of the constellation diagram, folding algorithm averages noise. Folding algorithm is applicable for higher order constellation
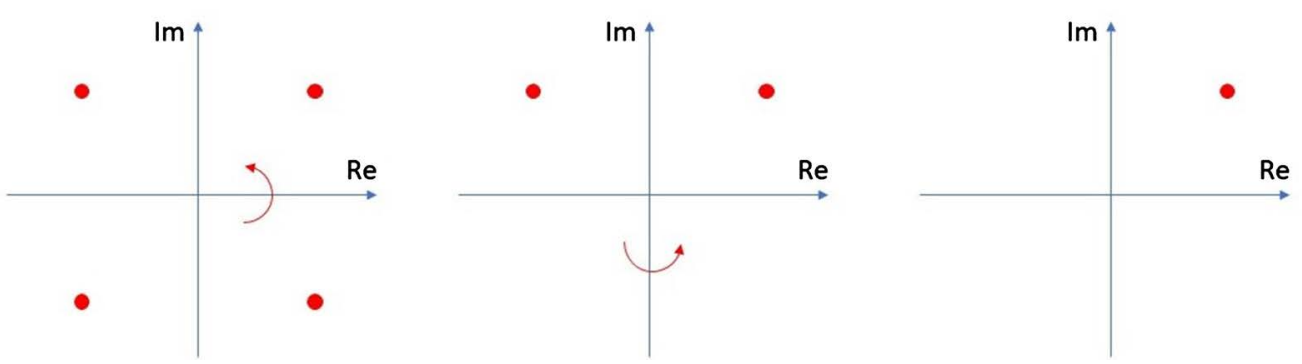

Figure 5. Explanation of the folding algorithm for 4QAM. Left: The original 4QAM. Middle: After a folding across the Re axis. Right: After a folding across the Im axis. Only one point remains, it is perfect for carrier recovery.

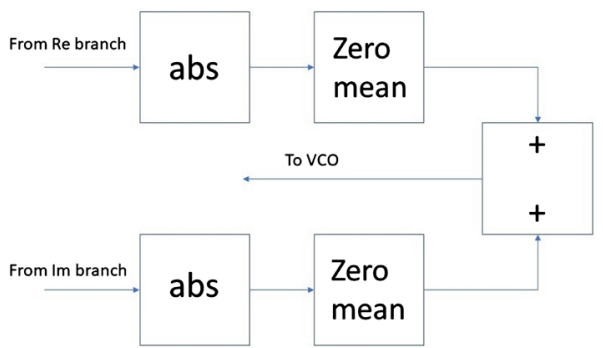

Figure 6. Part of the system realizing the folding algorithm. Folding itself is realized by applying absolute value as we have shown it in Figure 5.

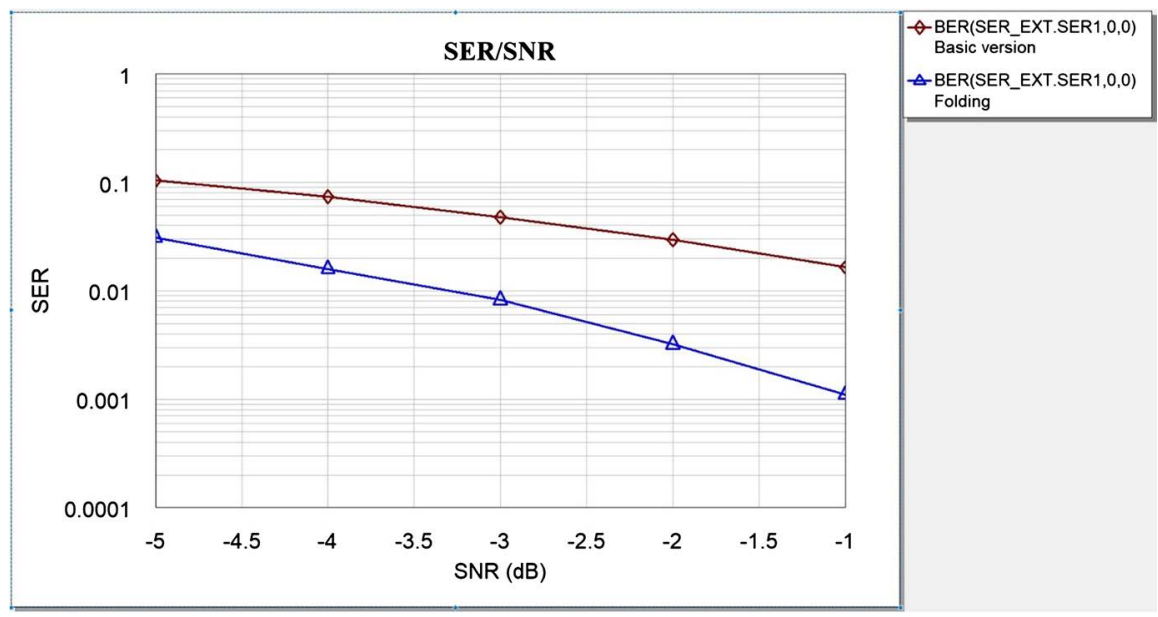

Figure 7. Result of the application of the folding algorithm. Upper curve: Basic Costas loop, lower curve: With folding algorithm. 
diagrams as well. We consider here 4QAM only.

\section{Application of the Rotational Average (Figures 8-10)}

Based on the right graph in Figure 5, a new idea occurs. The noise can also be averaged after folding algorithm, if the noise in the neighborhood of the remaining constellation point is rotated around the point. We try one 90 deg rotation, but the number of rotations can be arbitrary.
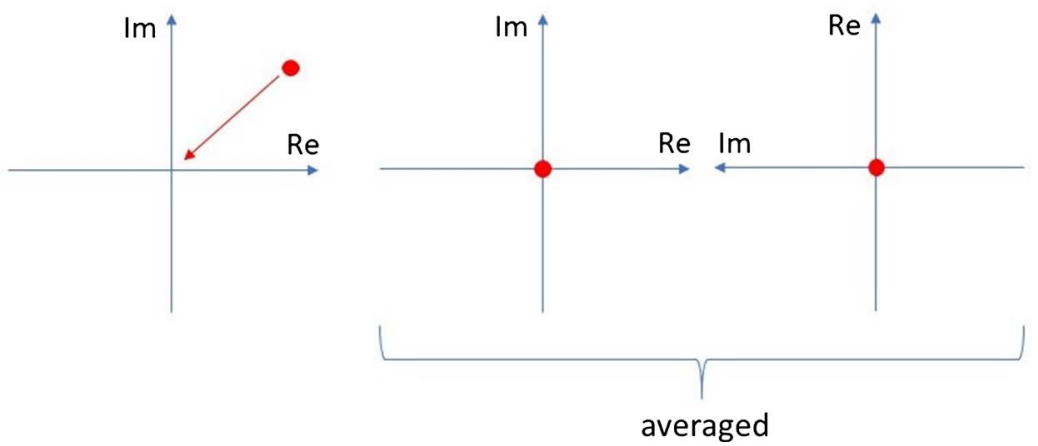

Figure 8. Sketch of application of rotational average.

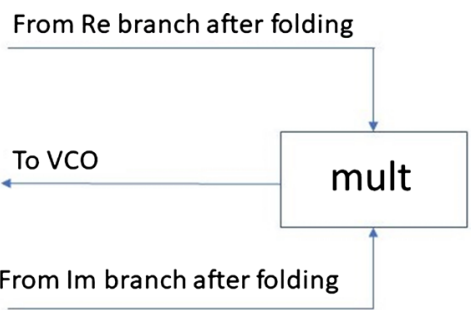

Figure 9. Part of the system realizing rotational average. Explanation: $\left(\frac{\mathrm{Re}+I m}{2}\right)^{2}-\left(\frac{\mathrm{Re}-I m}{2}\right)^{2}=\mathrm{Re} * I m$. Not $j I m$, this is not an error.

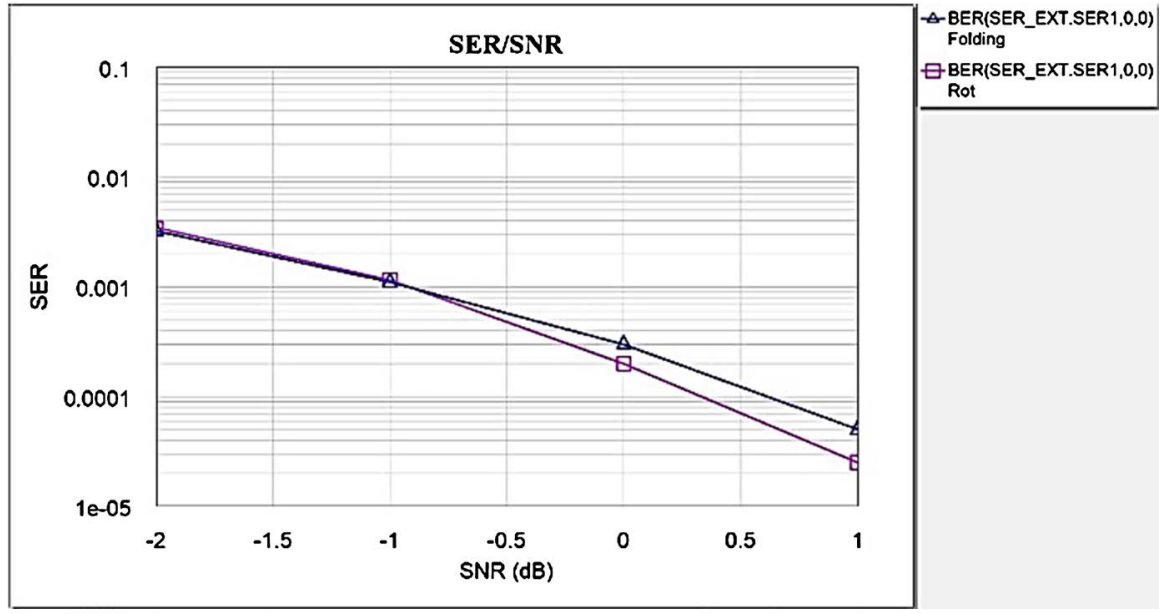

Figure 10. Result of application of rotational average. Upper curve: Folding algorithm alone, lower curve: With application of rotational average. For bad SNR, the two algorithms offer approximately the same performance. But at slightly better SNR, the advantage of the rotational average is obvious. 


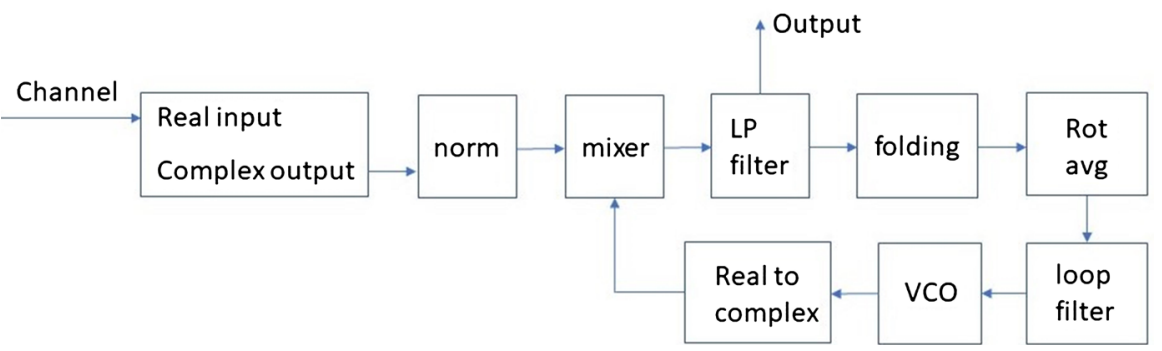

Figure 11. Block diagram of the improved Masterpiece.

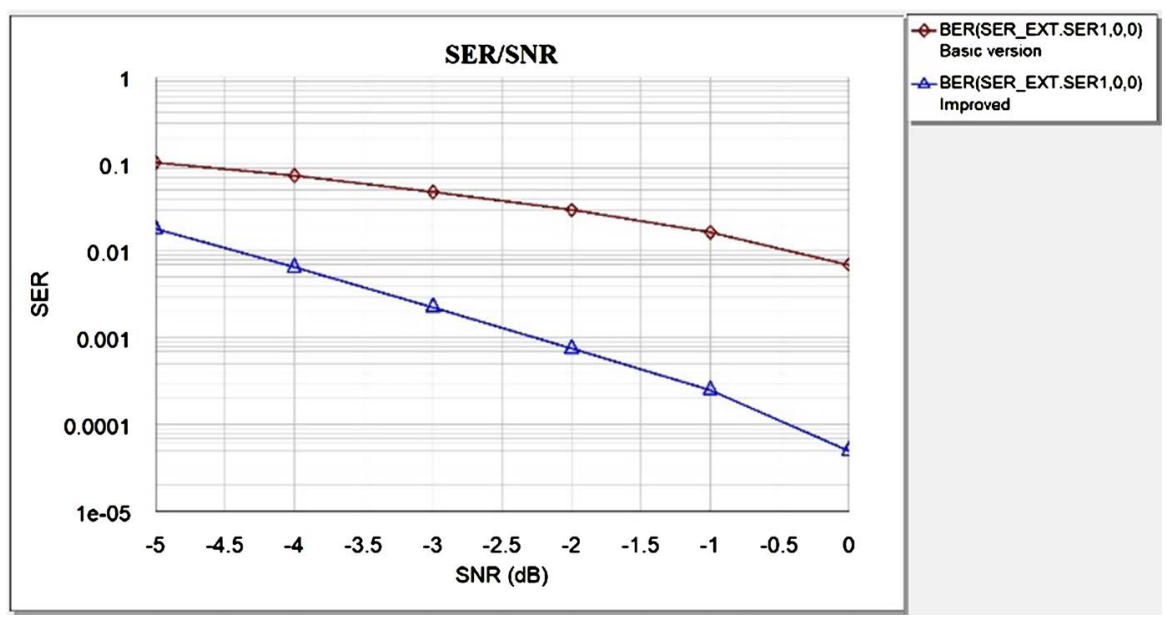

Figure 12. Noise properties of the improved Masterpiece compared to the basic Costas loop.

\section{The improved Masterpiece}

First, we show the schematics including complex signals with normalization, folding, and rotational average (Figure 11). Noise properties are shown in Figure 12.

\section{Conclusions}

In this paper an effective method for noise reduction for 4QAM communications has been shown. Other Hungarian efforts on decreasing the effect of interference and noise are found in [5]. Our intention is the application of this circuit in our version of quantum communications [6].

Email contact between the reader and the author is strongly recommended for providing repeatability of the results by sending the proper AWR files in case of interest.

Most recent results are, just before finishing this paper, that our Masterpiece can also work at SNR $=-22 \mathrm{~dB}$ and break the Shannon formula.

\section{Acknowledgements}

This research was started with a discussion with Dr. András Radványi at our sailing boat get together in Balaton Lake in August 2017. He put especially interesting questions, many thanks for them. 
Sincere thanks are due to Dr. Benedek Kovács, last colleague of the author at Ericsson Hungary before retirement, who made it possible for the author to work at home and he participated in this work as well as co-author of the patent [7].

\section{Conflicts of Interest}

The author declares no conflicts of interest regarding the publication of this paper.

\section{References}

[1] Proakis, J.G. (2001) Digital Communications. McGraw-Hill, New York.

[2] Best, R.E., Kuznetsov, N.V., Leonov, G.A., Yuldashev, M.V. and Yuldashev, R.V. (2016) Tutorial on Dynamic Analysis of the Costas Loop. Annual Reviews in Control, 42, 27-49. https://doi.org/10.1016/j.arcontrol.2016.08.003

[3] Ladvánszky, J. (2017) A Costas Loop with Differential Coding. International Journal of Contemporary Research and Review, 8, 20308-20314.

[4] Ladvánszky, J. (2018) A Costas Loop Variant for Large Noise. Journal of Asian Scientific Research, 8, 144-151. https://doi.org/10.18488/journal.2.2018.83.144.151

[5] Hilt, A. Maury, G. and Cabon, B. (1999) Radio-Frequency Interference in Digital Communication Links. Journal of Communications, 50, 36-43.

[6] Ladvánszky, J. Quantum Communications Are at Hand-The Hungarian Concept. In Press.

[7] Ladvánszky, J. and Kovács, B. (2018) Methods and Apparatus for Signal Demodulation. Ericsson Patent, 2018.03.01. P74032 WO1; PCT Application Date: 3/1/2018; Application Nr. PCT/SE2018/050198. 